Japan. J. Math.

Vol. 1, No. 2, 1975

\title{
On the index theorem for isometry-invariant geodesics
}

\author{
Takashi SAKAI \\ (Received January 13, 1975)
}

\section{§ 1. Introduction}

Let $(M,<,>)$ be a riemannian manifold, and $B \subset M \times M$ be a submanifold. Put $C_{B}:=\left\{\gamma:[a, b] \rightarrow M ; H^{1}\right.$-curves such that $\left.(\gamma(a), \gamma(b)) \in B\right\}$. We shall consider the energy integral $E(\gamma):=1 / 2 \int_{a}^{b}\langle\dot{\gamma}(t), \dot{\gamma}(t)\rangle d t$ on $C_{B}$. Then $c \in C_{B}$ is a critical point of $E(\gamma)$ if and only if $c:[a, b] \rightarrow M$ is a geodesic which satisfies the boundary condition $(c(a), c(b)) \in B,(\dot{c}(a),-\dot{c}(b)) \perp T_{(c(a), c(b))} B$. At a critical point $c \in C_{B}$, we may define the second variation $D^{2} E(c)$ which is a symmetric bilinear form on $T_{c} C_{B}=\left\{X:[a, b] \rightarrow c^{*} T M ; H^{1}\right.$-vector fields along $c$ such that $\left.(X(a), X(b)) \in T_{(c(a), c(b))} B\right\}$. Then the index of $c$ is defined as the dimension of a maximal subspace of $T_{c} C_{B}$ over which $D^{2} E(c)$ is negative definite. Geometrically, this indicates the number of essentially different curves in the neighbourhood of $c$ which are shorter than $c$ and satisfy the boundary condition.

Recently, using the intersection theory of curves in the manifold of Lagrange spaces of a symplectic vector space, J. J. Duistermaat ([3]) obtained the general index theorem for the geodesics in the category of calculus of variations. His theorem asserts that index of $c$ is expressed as the intersection number of the curve of Lagrange spaces $t \rightarrow \Phi(0, t)(a \leqq t \leqq b)$ with a fixed Lagrange space plus a correction term, where $\Phi(0, t)$ is the solution matrix of the linear Hamilton flow with eigenvalue parameter 0 .

On the other hand, for the typical cases $B=$ the diagonal set of $M \times M$ (i.e., closed geodesic case) or $B=N \times P(N, P \subset M$ submanifolds ; separate end manifolds case), the index theorem has been obtained directly, by constructing explicitly the Lagrange spaces of $V^{2(\operatorname{dim} M-1)}(\dot{c}(a))$ which is the orthogonal complement of the geodesic spray $\xi_{\dot{c}(a)}$ in $T_{\dot{c}(a)} U M$ (see [1], [4], [5], [6]). This approach uses more geometrical properties of Jacobi fields and doesn't appeal to the topological method.

In the present note we shall give the index theorem for the case $B=$ graph $f$, where $f$ is an isometry of $M$, by an explicit construction of the Lagrange space (i.e., index theorem for isometry-invariant geodesics). 
Index of isometry invariant geodesics appeared e.g. in a work of Colin de Verdière ([2]). This generalize a result of Klingenberg ([4], [5]), but the proof heavily depends on the Klingenberg's method. On the other hand, we shall treat the non-degenerate case and degenerate case in a unified manner.

\section{§ 2. Preliminaries}

2.1. Let $(M,<\rangle$,$) be an (n+1)$-dimensional riemannian manifold, and $p: T M \rightarrow M$ be the tangent bundle of $M$. Let $K: T T M \rightarrow T M$ denotes the connection map with respect to the Levi-Civita connection. Then for $X \in$ $T M$, we have an identification $T_{X} T M \approx T_{x} M \oplus T_{x} M(p(X)=x)$ via an isomorphism $\tilde{X}\left(\in T_{X} T M\right) \rightarrow\left(p_{*} X, K X\right)\left(\in T_{x} M \oplus T_{x} M\right)$. Under the above identification, we may write $\tilde{X}:=\left(X_{h}, X_{v}\right)$. In particular, the geodesic spray $\xi$ takes the form $\xi_{X}:=(X, 0)$ at $X \in T M$. We define the Sasaki metric $G$ on $T M$ by $G(\tilde{X}, \tilde{Y}):=\left\langle X_{h}, Y_{h}\right\rangle+\left\langle X_{v}, Y_{v}\right\rangle$.

Next let $U M$ be the differentiable manifold of unit tangent vectors to $M$. Then for $X \in U M$, we have an identification $T_{X} U M \approx T_{x} M \oplus \perp X(x=$ $p(X))$, where $\perp X:=\left\{Y \in T_{x} M ;\langle X, Y\rangle=0\right\}$. We shall denote by $V_{h}^{n+1}(X)$ (resp. $V_{v}^{n}(X)$ ) the first (resp. second) summand of the above identification of $T_{X} U M$. Let $V_{h}^{n}(X)$ be the orthogonal complement (with respect to $\langle$,$\rangle )$ of $X$ in $V_{h}^{n+1}(X)$, and we put $V^{2 n}(X):=V_{h}^{n}(X) \oplus V_{v}^{n}(X)$ which may be identified with the orthogonal complement (with respect to $G$ ) of the geodesic spray $\xi_{X}$ in $T_{X} U M$. Thus we have a vector bundle $\tau^{2 n}: V^{2 n} \rightarrow U M$.

Now, for every $X \in U M, V^{2 n}(X)$ has a natural symplectic structure $\alpha$ which is defined by $2 \alpha\left(\left(X_{h}, X_{v}\right),\left(Y_{h}, Y_{v}\right)\right):=\left\langle X_{h}, Y_{v}\right\rangle-\left\langle X_{v}, Y_{h}\right\rangle$. Then subspaces of $V^{2 n}(X)$ on which $\alpha$ vanishes identically will be called isotropic, and $n$-dimensional isotropic subspaces will be called Lagrange subspaces. For example, $V_{h}^{n}(X), V_{v}^{n}(X)$ are Lagrange subspaces.

2.2. Let $f:(M,\langle\rangle,) \rightarrow(M,\langle\rangle$,$) be an isometry. Then f$ induces an isometry $f_{*}:(U M, G) \rightarrow(U M, G)$, where $f_{*}$ is the differential of $f$. In fact, for $f_{* *}$ (differential of $f_{*}$ ): $T U M \rightarrow T U M$, we have $K f_{* *}=f_{*} K, p_{*} f_{* *}=f_{*} p_{*}$, and consequently

$$
f_{* *}\left(X_{h}, X_{v}\right)=\left(f_{*} X_{h}, f_{*} X_{v}\right) .
$$

Especially $f_{* *}: V^{2 n}(X) \rightarrow V^{2 n}\left(f_{*} X\right)$ preserves the sympletic form $\alpha$. easily

Next, we put $B=$ graph $f=\{(x, f(x)) \in M \times M ; x \in M\}$. Then we have

(2.2) $B$ is a totally geodesic submanifold with respect to the induced metric. 


$$
\left(T_{(x, f(x))} B\right)^{\perp}=\left\{(u, v) \in T_{x} M \oplus T_{f(x)} M ; f_{*} u+v=0\right\},
$$

where $\perp$ means the orthogonal complement.

2.3. In this subsection we shall review the Klingenberg's decomposition theorem of simpletic vector space via the Jordan canonical form of simpletic transformation ([4], [5]). Let $\left(V^{2 n}, \alpha\right)$ be a simpletic vector space and $P: V \rightarrow V$ be a simplectic transformation. We shall consider the complexification $V_{\boldsymbol{C}}$ of $V$, hermitian skew-symmetric extension of $\alpha$ (i.e., $\alpha(X, Y)$

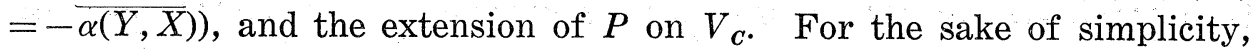
we shall denote these by the same notation $V, \alpha, P$. Let $V(\rho)$ be a generalized eigenspace of the eigenvalue $\rho$ of $P$. A sequence of vectors $\left\{x_{1}, \cdots, x_{k}\right\}$ will be called a $\rho$-sequence if $P x_{1}=\rho x_{1}, P x_{2}=\rho x_{2}+x_{1}, \cdots, P x_{k}=\rho x_{k}+x_{k-1}$ hold. Then, by Jordan's theorem, $V$ is a direct sum of $V(\rho)$ 's and each $V(\rho)$ has a basis consisting of $\rho$-sequences. When $P$ is sympletic, if $\rho$ is an eigenvalue of $P$, then $\bar{\rho}, \rho^{-1}, \bar{\rho}^{-1}$ are also eigenvalues of $P$. In this subsection 'orthogonal' means $\alpha$-orthogonal, and a subspace $W \subset V$ will be called non-degenerate if $\alpha_{\mid w}$ is non-degenerate. Then we have the following.

(2.4) $V$ is the orthogonal sum of $V(\rho) \oplus V\left(\bar{\rho}^{-1}\right) \quad(|\rho| \neq 1)$ and $V(\rho) \quad(|\rho|=1)$. $V(\rho)(|\rho|=1, \rho \neq \pm 1)$ has a basis consisting of mutually orthogonal nondegenerate $\rho$-sequences $\left\{x_{1}, \cdots, x_{k}\right\}$ with $\alpha\left(x_{i}, x_{j}\right)=0$ for $i+j \leqq k$ and $\alpha\left(x_{i}, x_{j}\right)$ $\neq 0$ for $i+j=k+1$. $\quad V( \pm 1)$ has a basis consisting of mutually orthogonal $\left\{e_{1}, \cdots, e_{2 k}\right\}$ 's and $\left\{f_{1}, \cdots, f_{2 k-1} ; \tilde{f}_{1}, \cdots, \tilde{f}_{2 k-1}\right\}$ 's, where $\left\{e_{1}, \cdots, e_{2 k}\right\}$ is a real non-degenerate $\rho$-sequence with $\alpha\left(e_{i}, e_{j}\right)=0$ for $i+j \leqq 2 k$ and $\alpha\left(e_{i}, e_{j}\right) \neq 0$ for $i+j=2 k+1$, and $\left\{f_{i} ; \tilde{f}_{j}\right\}$ is a non-degenerate subspace generated by real $\rho$ sequences $\left\{f_{i}\right\}$ and $\left\{\tilde{f}_{i}\right\}$ with odd elements $(\rho= \pm 1)$. Moreover $\left\{f_{i} ; \tilde{f}_{j}\right\}_{1 \leqq i, j \leqq k-1}$ is isotropic and $\left\{f_{i} ; \tilde{f}_{j}\right\}_{1 \leq i, j \leq k}$ is non-degenerate.

In the following, we shall fix these $\rho$-sequences which are determined up to the sympletic isomorphism.

(2.5) $\quad V^{2 n}=V_{c o} \oplus V_{n c}$ ： orthogonal decomposition of real non-degenerate invariant subspaces, where

$V_{n c}$ is generated by $V(\rho) \oplus V\left(\bar{\rho}^{-1}\right)(|\rho| \neq 1)$ and $\rho$-sequences $(|\rho|=1)$ which consists of more than one elements ;

$V_{c o}$ is generated by $\rho$-sequences $(|\rho|=1)$ with only one element.

Next, we shall define real non-degenerate subspaces $V_{i n}^{2 p}, V_{u n}^{2 q}(p+q=n)$ and real invariant isotropic subspace $V_{i n}^{p} \subset V_{i n}^{2 p}$. First for $\rho(|\rho| \neq 1), V(\rho)$ $\oplus V\left(\bar{\rho}^{-1}\right)$ belongs to $V_{i n}^{2 p}$, and isotropic $V(\rho)(|\rho|<1)$ belongs to $V_{i n}^{p}$. Next, for $\rho(|\rho|=1, \rho \neq \pm 1), \rho$-sequence $\left\{x_{1}, \cdots, x_{2 k}\right\}$ with even elements belongs to 
$V_{i n}^{2 p}$ and isotropic $\left\{x_{1}, \cdots, x_{k}\right\}$ belongs to $V_{i n}^{p}$. On the other hand, for $\rho$ sequence $\left\{x_{1}, \cdots, x_{2 k-1}\right\}$ with odd elements $(k>1)$, isotropic $\left\{x_{1}, \cdots, x_{k-1}\right\}$ belongs to $V_{i n}^{p}$. Since $\left\{x_{i}\right\}_{1 \leqq i \leqq k}$ is non-degenerate, we can take its orthogonal complement $\left\{x_{k+1}^{\prime}, \cdots, x_{2 k-1}^{\prime}\right\}$ in $\left\{x_{i}\right\}_{1 \leqq i \leqq 2 k-1}$. Then $\left\{x_{1}, \cdots, x_{k-1}, x_{k+1}^{\prime}, \cdots, x_{2 k-1}^{\prime}\right\}$ belongs to $V_{i n}^{2 p}$ and $\left\{x_{k}\right\}$ belongs to $V_{u n}^{2 q}$. Finally for $\rho= \pm 1, \rho$-sequence $\left\{e_{1}, \cdots, e_{2 k}\right\}$ with even elements belongs to $V_{i n}^{2 p}$ and isotropic $\left\{e_{1}, \cdots, e_{k}\right\}$ belongs to $V_{i n}^{p}$. For $\left\{f_{i} ; \tilde{f}_{j}\right\}_{1 \leqq i, j \leqq 2 k-1}(k>1),\left\{f_{i} ; \tilde{f}_{j}\right\}_{1 \leqq i, j \leqq k-1}$ belongs to $V_{i n}^{p}$. Since $\left\{f_{i} ; \tilde{f}_{j}\right\}_{1 \leqq i, j \leqq k}$ is non-degenerate we can take its orthogonal complement $\left\{f_{k+1}^{\prime}, \cdots, f_{2 k-1}^{\prime} ; \tilde{f}_{k+1}^{\prime}, \cdots, \tilde{f}_{2 k-1}^{\prime}\right\}$ in $\left\{f_{i} ; \tilde{f}_{j}\right\}_{1 \leqq i, j \leqq 2 k-1}$. Then $\left\{f_{1}, \cdots, f_{k-1}, f_{k+1}^{\prime}\right.$, $\left.\cdots, f_{2 k-1}^{\prime} ; \tilde{f}_{1}, \cdots, \tilde{f}_{k-1}, \tilde{f}_{k+1}^{\prime}, \cdots, \tilde{f}_{2 k-1}^{\prime}\right\}$ belongs to $V_{i n}^{2 p}$ and $\left\{f_{k}, \tilde{f}_{k}\right\}$ belongs to $V_{u n}^{2 q}$. On the other hand $V_{c o}$ is contained in $V_{u n}^{2 q}$. Thus we have,

(2.6) $V^{2 n}=V_{i n}^{2 p} \oplus V_{u n}^{2 q}$; orthogonal decomposition into real non-degenerate subspaces, where $V_{i n}^{2 p}$ has an real, invariant subspace $V_{i n}^{p}$.

\section{$\S 3$. Index theorem for isometry-invariant geodesics}

3.1. Let $B=\operatorname{graph} f$, where $f$ is an isometry of $(M,\langle\rangle$,$) . Let C_{B}$ := $\left\{\gamma:[a, b] \rightarrow M ; H^{1}\right.$-curve with $\left.(\gamma(a), \gamma(b)) \in B\right\}$ and $E(\gamma)$ be the energy integral on $C_{B}$. Then $c \in C_{B}$ is a critical point of $E(\gamma)$ if and only if $c$ is a geodesic which is invariant under $f$, i.e., $f(c(a))=c(b), f_{*}(\dot{c}(a))=\dot{c}(b)$ (c.f. $\S 1$ and (2.3)). Note that $c$ is complete, i.e., is defined for all values of parameter. At a critical point $c$ with $|\dot{c}(t)|=1$ for $t \in[a, b]$, we shall consider the second variation $D^{2} E(c)$. By (2.1) we have on $T_{c} C_{B}$,

$$
D^{2} E(c)(X(t), Y(t))=\int_{a}^{b}\{\langle\nabla X(t), \nabla Y(t)\rangle+\langle R(X(t), \dot{c}(t)) Y(t), \dot{c}(t)\rangle\} d t,
$$

where $\nabla$ denotes the covariant differentiation with respect to $\dot{c}(t)$, and $R$ denotes the curvature tensor. So, for the index problem it suffices to consider $D^{2} E(c)$ on the subspace

$$
\Xi=\left\{X(t) ; H^{1} \text {-vector fields along } c \text { such that } X(t) \perp \dot{c}(t) \text {, and } X(b)=f_{*} X(a)\right\} .
$$

That is, we have index of $\left.D^{2} E(c)\right|_{T_{c} C_{B}}=$ index of $\left.D^{2} E(c)\right|_{B}$.

3.2. Let $c:[a, b] \rightarrow M$ be a normal geodesic with $f(c(a))=c(b)$ and $f_{*}(\dot{c}(a))=\dot{c}(b)$. Then from $\S \S 2.1$, we have a vector bundle $V^{2 n}(t)=V_{h}^{n}(t) \oplus V_{v}^{n}(t)$ $\rightarrow[a, b]$ which is induced from $\tau^{2 n}: V^{2 n} \rightarrow U M$ via the map $\dot{c}:[a, b] \rightarrow U M$. Let $\varphi_{t}$ be the geodesic flow, then $\left(\varphi_{t}\right)_{*}: V^{2 n}\left(t_{0}\right) \rightarrow V^{2 n}\left(t+t_{0}\right) \operatorname{maps}(A, B) \in V^{2 n}\left(t_{0}\right)$ into $\left(Y\left(t+t_{0}\right), \nabla Y\left(t+t_{0}\right)\right)$, where $Y$ is a Jacobi field with $Y\left(t_{0}\right)=A, \nabla Y\left(t_{0}\right)=B$. In the following we shall use the notation $\tilde{Y}(t):=(Y(t), \nabla Y(t))$ for Jacobi field $Y(t)$. 
Now, if we define $P_{f}:=f_{* *}^{-1}\left(\varphi_{b_{-}}\right)_{*}$, then since geodesic flow respects $\alpha$ and $(2,1)$ hold, $P_{f}$ is a linear symplectic transformation of $\left(V^{2 n}(\alpha), \alpha\right)$. Applying $\S \S 2.3$ to this symplectic transformation $P_{f}$, we get the $(\alpha-)$ orthogonal decomposition $V^{2 n}(a)=V_{i n}^{2 p} \oplus V_{i n}^{2 q}$, where $V_{i n}^{2 p}$ has an isotropic subspace $V_{i n}^{p}$. Then for the Lagrange space $V_{v}^{n}(a)$, the projection $V_{v}^{n}(a) \cap\left(V_{i n}^{p} \oplus V_{u n}^{2 q}\right)$ $\rightarrow V_{u n}^{2 q}$ modulo $V_{i n}^{p}$ determines an isotropic subspace $V_{u n}^{q} \subset V_{u n}^{2 q}$ (see [3], Lemma 2.3.). We define the Lagrange space $V_{e}^{n}=V_{i n}^{p} \oplus V_{u n}^{q}$. Then $V_{e}^{n}$ is also decomposed as a direct sum $V_{e}^{n}=V_{i n}^{p} \oplus V_{v}^{q}\left(V_{v}^{q} \subset V_{v}^{n}(a)\right)$.

3.3. Let $V_{1}$ be the real even dimensional non-degenerate subspace of $V_{c o}$ which is generated by the 1-sequences consisting of one element. Let $V_{\overline{1}}$ be the orthogonal complement of $V_{1}$, and $\pi: V_{e}^{n} \rightarrow V_{1}$ be the projection modulo $V_{\overline{1}}$. Note that $V_{i n}^{p}$ is a subspace of $\mathscr{V}:=\operatorname{Ker} \pi=V_{\overline{1}} \cap V_{e}^{n}$. We take $\mathscr{U} \subset V_{v}^{q}$ as a complement to $\mathscr{V}$ in $V_{e}^{n}$, and put $u(\tilde{Y}):=\left(\left.\pi\right|_{\mathscr{U}}\right)^{-1} \pi(\tilde{Y})$. We shall see that the index theorem turns out to be independent of the choice of $\mathscr{U}$. Then clearly $V_{e}^{n}=\mathscr{U} \oplus \mathscr{V} . \mathscr{V}$ may be decomposed into the form $\mathscr{V}=V_{i n}^{p} \oplus \mathscr{V} u n$ $\left(\mathscr{V}_{u n} \subset V_{u n}^{q}\right)$. Finally we put $v:=i d-u$. Then $v\left(V_{e}^{n}\right)=\mathscr{V}$.

3.4. Now we shall define the notion of conjugate points. Put $W(t)$ : $=\left(\varphi_{t-a}\right)_{*} V_{e}^{n} \cap V_{v}^{n}(t)$. First we define for $a<t_{0}<b$,

$C\left(t_{0}\right):=$ vector space of once broken Jacobi fields $Z(t)$ defined by $Z(t)=$ $Z_{1}(t), a \leqq t \leqq t_{0} ; Z(t)=Z_{2}(t), t_{0} \leqq t \leqq b$, where $Z_{1}(t), Z_{2}(t)$ are Jacobi fields such that

(i ) $\tilde{Z}_{1}\left(t_{0}\right)-\tilde{Z}_{2}\left(t_{0}\right)\left(=\tilde{Z}\left(t_{0}-0\right)-\tilde{Z}\left(t_{0}+0\right)\right)=: \tilde{Y}\left(t_{0}\right) \in W\left(t_{0}\right)$

(ii) $\quad\left(P_{f}-i d\right) \tilde{Z}_{1}(t)=\left(P_{f}-i d\right) \tilde{Y}(t)+(v \tilde{Y}(a))(t)$ for $a \leqq t \leqq t_{0}$.

REMARK 1. From (i), (ii), we have

(iii) $\quad\left(p_{f}-i d\right) \tilde{Z}_{2}(t)=(v \tilde{Y}(a))(t)$ for $t_{0} \leqq t \leqq b$,

and $C\left(t_{0}\right) \subset \Xi$. In fact, $\left(Z(a)-f_{*}^{-1} Z(b), \nabla Z(a)-f_{*}^{-1} \nabla Z(b)\right)=\tilde{Z}_{1}(a)-f_{* *}^{-1} \tilde{Z}_{2}(b)=$ $\tilde{Z}_{1}(a)-P_{f} \tilde{Z}_{2}(a)=\tilde{Z}_{1}(a)-\tilde{Z}_{2}(a)-\left(P_{f}-i d\right) \tilde{Z}_{2}(a)=\tilde{Y}(a)-v \tilde{Y}(a)=u \tilde{Y}(a) \in V_{v}^{q}$.

REMARK 2. Let $\mathfrak{R}:=\left\{Y(t)\right.$; Jacobi field with $\left.f_{* *} \tilde{Y}(a)=\tilde{Y}(b)\right\}$ be the null space of $D^{2} E(c)$ on $\Xi$. Then obviously $\mathfrak{R} \subset C\left(t_{0}\right)$.

Definition. Put $\bar{n}\left(t_{0}\right):=\operatorname{dim}\left(C\left(t_{0}\right) / \mathfrak{R}\right)$. We say that $t_{0}$ is a conjugate point if $\bar{n}\left(t_{0}\right)$ is positive and $\bar{n}\left(t_{0}\right)$ will be called the multiplicity of the conjugate point $t_{0}$.

Similary we define

$C(a):=$ vector space of Jacobi fields $Z(t)$ such that $\left(P_{f}-i d\right) \tilde{Z}(a)=v \tilde{Y}(a)$ for some $\tilde{Y}(\alpha) \in W(a)$. 
Then it is easy to see $C(a) \subset \Xi$ and $\mathfrak{R} \subset C(a)$. Note that $\mathscr{U} \subset W(a)$ holds. Now the following lemma is well-known.

LEMMA 1 ([4], [5]). Let $n_{0}:=n\left(t_{0}\right)=\operatorname{dim} W\left(t_{0}\right) \neq\{0\}$. Choose a basis $\left\{\tilde{Y}_{j}(t)=\left(Y_{j}(t), \nabla Y_{j}(t)\right)\right\}_{1 \leqq j \leqq n}$ for $\left(\varphi_{t-a}\right)_{*} V_{e}^{u}$ such that $\left\{\tilde{Y}_{j}\left(t_{0}\right)\right\}_{1 \leqq j \leqq n_{0}}$ is a basis for $W\left(t_{0}\right)$. Then we have,

(i ) $\nabla Y_{i}\left(t_{0}\right), 1 \leqq i \leqq n_{0} ; Y_{j}\left(t_{0}\right), n_{0}+1 \leqq j \leqq n$ form a basis for $\perp \dot{c}\left(t_{0}\right)$.

(ii) For $t \neq t_{0}$ sufficiently near $t_{0},\left\{Y_{j}(t)\right\}_{1 \leqq j \leqq n}$ are linearly independent. So $W\left(t_{0}\right) \neq\{0\}$ only for finite values of $t$.

Next, note that $C\left(t_{0}\right) \cap C\left(t_{0}^{\prime}\right)=\mathfrak{R}\left(a \leqq t_{0}<t_{0}^{\prime}<b\right)$. Then we have

LEMMA 2. (i) $\zeta: \oplus_{a<t_{0}<b} C\left(t_{0}\right) \rightarrow \oplus_{a<t_{0}<b} W\left(t_{0}\right)$ which maps $Z(t) \in C\left(t_{0}\right)$ into $\tilde{Z}_{1}\left(t_{0}\right)-\tilde{Z}_{2}\left(t_{0}\right)=\tilde{Y}\left(t_{0}\right) \in W\left(t_{0}\right)$ is a surjective homomorphism with kernel R. (ii) $\zeta_{a}: C(\alpha) \rightarrow v W(a)$ which maps $Z(t) \in C(a)$ into $v(\tilde{Y}(a))$ is a surjective homomorphism with kernel $\mathfrak{N}$.

Proof. $\zeta$ and $\zeta_{a}$ are surjective because $v W(a) \subset \mathscr{V}$ is contained in $\left(P_{f}-i d\right) V^{2 n}(a)$.

REMARK 3. Since $\left.\zeta\right|_{C\left(t_{0}\right)}: C\left(t_{0}\right) \rightarrow W\left(t_{0}\right)$ is a surjective linear map with kernel $\mathfrak{n}$, we have $\bar{n}\left(t_{0}\right)=n\left(t_{0}\right)$. By lemma $1, \bar{n}\left(t_{0}\right)$ is positive only for finite values of $t_{0}$.

3.5. Now we define $\lambda_{f}: \oplus_{a \leqq t_{0}<b} C\left(t_{0}\right) \rightarrow \mathscr{V}_{\text {un }}$ by $\lambda_{f} Z(t):=(v((\zeta Z)(a)))_{u n}$ for $Z(t) \in \oplus_{a \leqq t_{0}<b} C\left(t_{0}\right)$ and $\lambda_{f} Z(t):=\left(\zeta_{a} Z\right)_{u n}$ for $Z(t) \in C(\alpha)$. Then we get,

LEMMA 3. $\quad \lambda_{f}: \oplus_{a \leqq t_{0}<b} C\left(t_{0}\right) \rightarrow \mathscr{V}_{u n}$ is a surjective map and we have

$$
\operatorname{dim} \operatorname{Ker} \lambda_{f}=\sum_{a<t_{0}<b} \bar{n}\left(t_{0}\right)+\operatorname{dim}\left(V_{v}^{n}(a) \cap V_{i n}^{p}\right)+\operatorname{dim} \Re .
$$

Proof. Since $\mathscr{V}_{u n} \subset V_{i n}^{p} \oplus V_{v}^{q}$, for any $\tilde{Y}_{u n} \in \mathscr{V}_{u n}$, there exist $\tilde{Y}_{i n} \in V_{i n}^{p}$ and $\tilde{Y}_{q} \in V_{v}^{q}$ such that $\tilde{Y}_{u n}=\tilde{Y}_{i n}+\tilde{Y}_{q}$. Then $\tilde{Y}_{q} \in W(a)$. Now the surjectivity of $\lambda_{f}$ follows from the surjectivity of $\zeta$ and $\zeta_{a}$. Next we shall define a linear map $\Phi: \mathscr{U} \oplus \mathscr{V}_{u n} \oplus\left(V_{v}^{n}(\alpha) \cap V_{i n}^{p}\right)$ (direct sum) $\rightarrow W(\alpha)$ by $\left(\tilde{Y}_{\mathscr{q}}, \tilde{Y}_{u n}=\tilde{Y}_{i n}\right.$ $\left.+\tilde{Y}_{q}, \tilde{Y}_{n}\right) \rightarrow \tilde{Y}_{\mathscr{U}}+\tilde{Y}_{q}+\tilde{Y}_{n} \in W(a)$. Then clearly $\Phi$ is injective. On the other hand, since $W(a)=\mathscr{U} \oplus W(a) \cap \mathscr{V}$ holds and $\left.\Phi\right|_{\mathscr{V}_{u n} \oplus\left(V_{v}^{n}(a) \cap V_{i n}^{p}\right)}$ is a surjective map onto $W(a) \cap \mathscr{V}$ by the same reason as above, we see that $\Phi$ is surjective. So $\operatorname{dim} W(a)=\operatorname{dim} \mathscr{U}+\operatorname{dim} \mathscr{V}_{u n}+\operatorname{dim}\left(V_{v}^{n}(a) \cap V_{i n}^{p}\right)$. Now,

$$
\begin{aligned}
& \operatorname{dim} \operatorname{Ker} \lambda_{f}=\operatorname{dim} \bigoplus_{a \leqq t_{0}<b} C\left(t_{0}\right)-\operatorname{dim} \mathscr{V}_{u n}=\sum_{a<t_{0}<b} \operatorname{dim} W\left(t_{0}\right)+\operatorname{dim} v W(a) \\
& +\operatorname{dim} \mathfrak{N}-\operatorname{dim} \mathscr{V}_{u n}=\sum_{a<t_{0}<b} \operatorname{dim} W\left(t_{0}\right)+\operatorname{dim} W(a)+\operatorname{dim} \mathfrak{N} \\
& -\operatorname{dim} \mathscr{U}-\operatorname{dim} \mathscr{V}_{u n}=\sum_{a<t_{0}<b} \operatorname{dim} W\left(t_{0}\right)+\operatorname{dim}\left(V_{v}^{n}(a) \cap V_{i n}^{p}\right) \\
& +\operatorname{dim} \mathfrak{\Re}
\end{aligned}
$$


Next we define a bilinear form $Q_{f}$ on $\mathscr{V}_{u n}$ by $Q_{f}\left(\tilde{Y}(a), \tilde{Y}^{\prime}(a)\right):=$ $-2 \alpha\left(\tilde{Y}(a),\left(P_{f}-i d\right)^{-1} \tilde{Y}^{\prime}(a)\right)$ for $\tilde{Y}(a), \tilde{Y}^{\prime}(a) \in \mathscr{V}_{u n} . \quad$ Note that $\left(P_{f}-i d\right)^{-1} \tilde{Y}^{\prime}(a)$ is determined modulo eigenvectors in $V_{i n}^{p}$ of eigenvalues 1 and eigenvectors in $V_{1}$ which are $(\alpha-)$ orthogonal to $\mathscr{V}_{u n}$, so $Q_{f}$ is well-defined. $Q_{f}$ is a symmetric bilinear form (c.f. (4.1)).

Now we shall state our theorem.

THEOREM. Index $c\left(:=\right.$ index of $D^{2} E(c)$ on $\left.T_{c} C_{B}\right)=\sum_{a<t_{0}<b} \bar{n}\left(t_{0}\right)+$ $\operatorname{dim}\left(V_{v}^{n}(a) \cap V_{i n}^{p}\right)+($ index + nullity $) Q_{f}$.

\section{§ 4. Proof of the theorem}

4.1. Let $W\left(t_{0}\right) \neq\{0\}$. Then by lemma 2 , for $\tilde{Y}\left(t_{0}\right) \in W\left(t_{0}\right)$ we have an element $Z(t) \in \zeta^{-1}\left(\tilde{Y}\left(t_{0}\right)\right) \subset \oplus_{a \leqq t<b} C\left(t_{0}\right)$ which is determined modulo $\Re$. Then, for $Z(t) \in \zeta^{-1}\left(\tilde{Y}\left(t_{0}\right)\right), Z^{\prime}(t) \in \zeta^{-1}\left(Y^{\prime}\left(t_{0}^{\prime}\right)\right)\left(t_{0} \geqq t_{0}^{\prime}\right)$, we have

$$
D^{2} E(c)\left(Z, Z^{\prime}\right)=Q_{f}\left(\lambda_{f} Z, \lambda_{f} Z^{\prime}\right)
$$

In fact, for e.g. $a<t_{0}^{\prime} \leqq t_{0}<b$,

$$
\begin{aligned}
D^{2} E(c)\left(Z, Z^{\prime}\right) & =\int_{a}^{b}\left\{\left\langle\nabla Z(t), \nabla Z^{\prime}(t)\right\rangle-\left\langle R(Z(t), \dot{c}(t)) \dot{c}(t), Z^{\prime}(t)\right\rangle\right\} d t \\
& =\left\langle\nabla Z(b), Z^{\prime}(b)\right\rangle-\left\langle\nabla Z(a), Z^{\prime}(a)\right\rangle+\left\langle\nabla Z\left(t_{0}-0\right)-\nabla Z\left(t_{0}+0\right), Z^{\prime}\left(t_{0}\right)\right\rangle \\
& =\left\langle f_{*}^{-1} \nabla Z_{2}(b)-\nabla Z_{1}(a), Z^{\prime}(a)\right\rangle+\left\langle\nabla Y\left(t_{0}\right), Z^{\prime}\left(t_{0}\right)\right\rangle \\
& =-2\left\{\alpha\left(\left(P_{f}-i d\right) \tilde{Z}_{2}(a)+\tilde{Z}_{2}(a)-\tilde{Z}_{1}(a), \tilde{Z}_{1}^{\prime}(a)\right)+\alpha\left(\tilde{Y}\left(t_{0}\right), \tilde{Z}_{2}^{\prime}\left(t_{0}\right)\right)\right\} \\
& =-2\left\{\alpha\left(v \tilde{Y}(a)-\tilde{Y}(a), \tilde{Z}_{1}^{\prime}(a)\right)+\alpha\left(\tilde{Y}(a), \tilde{Z}_{1}^{\prime}(a)-\tilde{Y}^{\prime}(a)\right)\right\} \\
& =-2 \alpha\left(v \tilde{Y}(a), \tilde{Z}_{1}^{\prime}(a)\right)=-2 \alpha\left(v \tilde{Y}(a),\left(P_{f}-i d\right)^{-1} v \tilde{Y}^{\prime}(a)\right) \\
& =-2 \alpha\left((v \tilde{Y}(a))_{u n},\left(P_{f}-i d\right)^{-1}\left(v \tilde{Y}^{\prime}(a)\right)_{u n}\right) \\
& \left.=Q_{f}(v \tilde{Y}(a))_{u n},\left(v \tilde{Y}^{\prime}(a)\right)_{u n}\right) \\
& =Q_{f}\left(\lambda_{f} Z, \lambda_{f} Z^{\prime}\right)
\end{aligned}
$$

In the above equalities, we have used the facts that $V_{e}^{n}$ is isotropic, $\alpha\left(V_{i n}^{p}, \mathscr{V}_{u n}\right)$ $=0$, and $\left(P_{f}-i d\right)$ leaves $V_{i n}^{p}$ invariant.

So there exists a subspace $\mathscr{L} \subset \oplus_{a \leqq t<b} C(t)$, with $\mathscr{L} \cap \Re=\{0\}$ such that its dimension is equal to $\operatorname{dim} \lambda_{f}^{-1}\left(\right.$ Null space of $\left.Q_{f}\right)-\operatorname{dim} \Re$ and $D^{2} E(c) \mid \mathscr{L} \equiv 0$. On the other hand, there is a subspace $\mathscr{L}^{\prime} \subset \oplus_{a \leqq t<b} C(t)$, which is linearly independent to $\operatorname{Ker} \lambda_{f}$, is of dimension equal to index $Q_{f}$, and such that $D^{2} E(c) \mid \mathscr{L}^{\prime}$ is negative definite. Since $\mathscr{L}^{\prime}$ and the negative part of $\mathscr{L}$ are independent, we get 
(4.2) Index of $\left.D^{2} E(c)\right|_{s} \geqq \operatorname{dim} \mathscr{L}+\operatorname{dim} \mathscr{L}^{\prime}$

$$
\begin{aligned}
& =\operatorname{dim} \operatorname{Ker} \lambda_{f}-\operatorname{dim} \Re+(\text { nullity }+ \text { index }) Q_{f} \\
& =\sum_{a<t<b} \operatorname{dim} W(t)+\operatorname{dim}\left(V_{v}^{n}(a) \cap V_{i n}^{p}\right)+(\text { nullity }+ \text { index }) Q_{f},
\end{aligned}
$$

by virtue of lemma 3 .

4.2. To see that the inverse inequality does hold, from the definition of $\mathscr{L}$ and $\mathscr{L}^{\prime}$, it suffices to show the following:

$$
\text { If a differentiable } \xi \in E \text { satisfies } D^{2} E(c)\left(\xi, \oplus_{a \leqq t<b} C(t)\right)=0 \text {, }
$$

then $D^{2} E(c)(\xi, \xi) \geqq 0$.

To show this let $Z(t) \in \zeta^{-1}\left(\tilde{Y}\left(t_{0}\right)\right) \subset C\left(t_{0}\right)\left(a<t_{0}<b\right)$. Then we get

$$
D^{2} E(c)(Z, \xi)=\int_{a}^{b} d / d t\langle\nabla Z(t), \xi(t)\rangle d t=\left\langle\nabla Y\left(t_{0}\right), \xi\left(t_{0}\right)\right\rangle-\langle\nabla u Y(a), \xi(a)\rangle,
$$

where $u Y(t)$ denotes the Jacobi field defined by $(u Y(a), \nabla u Y(a))=u(\tilde{Y}(a))$. Similarly, for $Z(t) \in \zeta_{a}^{-1}(v \tilde{Y}(a)) \subset C(a)$, we get

$$
D^{2} E(c)(Z, \xi)=\langle\nabla Y(a), \xi(a)\rangle-\langle\nabla u Y(a), \xi(a)\rangle .
$$

Now $u(\tilde{Y}(a)) \in \mathscr{U} \rightarrow\langle\nabla u Y(a), \xi(a)\rangle$ is a linear form on $\mathscr{U}$. Since the subspace generated by $\mathscr{U}$ and $V_{1}$ is non-degenerate and $\alpha \mid \mathscr{U} \equiv 0$, the above linear form may be written in the form $u(\tilde{Y}(a)) \in \mathscr{U} \rightarrow 2 \alpha\left(u(\tilde{Y}(a)), \tilde{Y}_{1}\right)=-\langle\nabla u Y(a)$, $\left.Y_{1}(a)\right\rangle$ for some $\tilde{Y}_{1} \in V_{1}$. Then if we put $\xi_{1}=\xi+Y_{1}$, where $Y_{1}$ belongs to $\mathfrak{R}$, we get

$$
D^{2} E(c)\left(Z, \xi_{1}\right)=\left\langle\nabla Y\left(t_{0}\right), \xi_{1}\left(t_{0}\right)\right\rangle=-2 \alpha\left(v(\tilde{Y}(a)), \tilde{Y}_{1}(a)\right)=0 \quad\left(a \leqq t_{0}<b\right) .
$$

Next we choose a basis $\left\{\tilde{Y}_{i}(\alpha)\right\}_{1 \leqq i \leqq n}$ such that $\left\{\tilde{Y}_{i}(a)\right\}_{1 \leqq i \leqq p}$ forms a basis of $V_{i n}^{p}$ and $\left\{\tilde{Y}_{\alpha}(a)\right\}_{p+1 \leqq \alpha \leqq n}$ forms a basis for $V_{u n}^{q}$. Since $\left\{P_{f} \tilde{Y}_{j}(a)=f_{* *}^{-1} \tilde{Y}_{j}(b)\right\}_{1 \leqq j \leqq p}$ generates $P_{f} V_{i n}^{p}=V_{i n}^{p}$, we have

$$
f_{* *}^{-1} \tilde{Y}_{j}(b)=\sum_{k=1}^{p} a_{j}^{k} \tilde{Y}_{k}(a) \quad(j=1, \cdots, p),
$$

where $\left(a_{j}^{k}\right)$ is non-singular. Then from (4.4), lemma $1, \xi_{1}(b)=f_{*} \xi_{1}(a)$, and (4.5), we know that $\xi_{1}(t)$ may be expressed in the form $\xi_{1}(t)=\sum_{i=1}^{n} w_{i}(t) Y_{i}(t)$ with differentiable $w^{i}(t)$.

Now from $\xi_{1}(b)=f_{*} \xi_{1}(a)$, we get $\sum_{\beta=p+1}^{n} w^{\beta}(b) Y_{\beta}(b)=\sum_{k=1}^{p}\left\{\sum_{j=1}^{p} w^{j}(a) b_{j}^{k}\right.$ $\left.-w^{k}(b)\right\} Y_{k}(b)$, where $\left(b_{j}^{i}\right)$ denotes the inverse matrix of $\left(a_{j}^{i}\right)$, i.e.,

$$
\sum_{\beta=p+1}^{n} w^{\beta}(b) \tilde{Y}_{\beta}(b)-\sum_{k=1}^{p}\left(\sum_{j=1}^{p} w^{j}(a) b_{j}^{k}-w^{k}(b)\right) \tilde{Y}_{k}(b)=\tilde{U}(b) \in W(b) .
$$

Then, by direct calculation using this and (4.5) we can show that 


$$
\left\langle\sum_{r=1}^{n} w^{r}(b) \nabla Y_{r}(b), \sum_{s=1}^{n} w^{s}(b) Y_{s}(b)\right\rangle-\left\langle\sum_{r=1}^{n} w^{r}(a) \nabla Y_{r}(a), \sum_{s=1}^{n} w^{s}(a) Y_{s}(a)\right\rangle
$$

$=0$ does hold. Finally we have

$$
\begin{aligned}
D^{2} E(c)(\xi, \xi) & =D^{2} E(c)\left(\xi_{1}, \xi_{1}\right) \\
& =\int_{a}^{b}\left|\sum \dot{w}^{j}(t) Y_{j}(t)\right|^{2} d t+\int_{a}^{b} d / d t\left\langle\sum_{k=1}^{n} w^{k}(t) \nabla Y_{k}(t), \xi(t)\right\rangle d t \\
& \geqq\left\langle\sum_{r=1}^{n} w^{r}(b) \nabla Y_{r}(b), \sum_{s=1}^{n} w^{s}(b) Y_{s}(b)\right\rangle \\
& -\left\langle\sum_{r=1}^{n} w^{r}(a) \nabla Y_{r}(a), \sum_{s=1}^{n} w^{s}(a) Y_{s}(a)\right\rangle=0 .
\end{aligned}
$$

This completes the proof of theorem.

REMARK 4. If $c$ is non-degenerate (i.e., 1 is not an eigenvalue of $P_{f}$ ), then $\mathscr{V}=V_{e}^{n}$ and $\mathscr{V}_{u n}=V_{u n}^{q}$.

REMARK 5. If $P_{f}=$ identity, then $V_{e}^{n}=V_{v}^{n}(a), V_{i n}^{p}=\{0\}$, and $\lambda_{f}=0$. So Index $c=\sum_{a<t<b} \operatorname{dim}\left(\left(\varphi_{t-a}\right)_{*} V_{v}^{n}(a)\right) \cap V_{v}^{n}(t)=$ sum of the multiplicities of ordinary conjugate points.

\section{References}

[1] W. Ambrose: The index theorem in Riemannian geometry, Ann. of Math., 73 (1961), 49-86.

[2] Y. Colin de Verdière: Spectre du laplacien et longueurs des géodésiques périodiques II, Compositio Math., 27 (1973), 159-184.

[ 3 ] J. J. Duistermaat: On the Morse index in variational calculus. Preprint.

[4] W. Klingenberg: The index theorem for closed geodesics, Tôhoku Math. J., 26 (1974), 573-580.

[ 5 ] — - Der Indexsatz für geschlossene Geodätische, Math. Z., 139 (1974), 231 256.

[6 ] T. Sakai: On the index theorem of Ambrose, Hokkaido Math. J., 4 (1975), 227233.

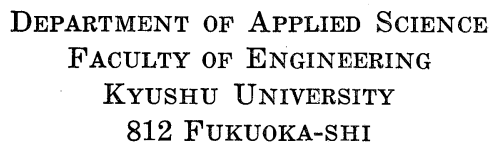

